Cahiers de philosophie de l'université de

Levinas : au-delà du visible

\title{
De la « signification corporelle du temps »
}

\section{Gérard Bensussan}

\section{(2) OpenEdition}

\section{Journals}

Édition électronique

URL : https://journals.openedition.org/cpuc/917

DOI : $10.4000 /$ cpuc. 917

ISSN : 2677-6529

\section{Éditeur}

Presses universitaires de Caen

\section{Édition imprimée}

Date de publication : 31 juillet 2012

Pagination : 315-324

ISBN : 978-2-84133-410-0

ISSN : 1282-6545

Référence électronique

Gérard Bensussan, «De la « signification corporelle du temps » , Cahiers de philosophie de l'université de Caen [En ligne], 49 | 2012, mis en ligne le 07 juin 2018, consulté le 02 février 2023. URL : http:// journals.openedition.org/cpuc/917; DOI : https://doi.org/10.4000/cpuc.917

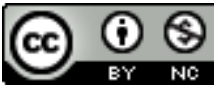

Creative Commons - Attribution - Pas d'Utilisation Commerciale 4.0 International - CC BY-NC 4.0 https://creativecommons.org/licenses/by-nc/4.0/ 


\section{De la «signification corporelle du temps»}

\section{J}

E VOUDRAIS PARTIR D'UNE EXPRESSION qu'on trouve dans les Carnets de captivité, rapidement et nerveusement notée au détour d'une annotation ou plus exactement en guise de sceau conclusif d'une dizaine de lignes du carnet 7. Cette expression, cette quasi-apostille, a de quoi provoquer à penser : "signification corporelle du temps ${ }^{1}$. Je reviendrai sur les neuf lignes qui la précèdent et lui donnent de quelque façon sa signification propre ou en tout cas son cadre et son champ.

La formule fait résonner quelque chose du geste lévinassien qui culminera dans Totalité et Infini, à savoir un renversement ou une inversion de l'intentionnalité, et dans une moindre mesure de la constitution, mais cette double opération relève d'un unique mouvement pour Levinas. «Signification corporelle du temps» se lit d'emblée, me semble-t-il, comme une formule, un chiffre - quelque chose qui voudrait régler en profondeur un certain rapport à la phénoménologie husserlienne. Disons, un peu massivement pour l'instant, que ce lien du temps et du corps vient à Levinas de sa lecture de Husserl - et d'une certaine signification temporelle du corps qu'on y trouverait sans mal. Quelques mots rapides, donc, pour commencer, de cet héritage husserlien.

1. Mon corps : parmi tous les corps qui sont là pour moi, je n'ai d'expérience originaire que de mon corps. Le premier phénomène à se présenter pour moi, en effet, c'est mon corps. C'est par lui, comme Leib, que me viennent toutes les sensations qui sont les miennes, olfactives, tactiles, auditives, perceptives en général. C'est par ce corps charnel dont j'ai l'expérience immédiate que je peux avoir toutes sortes de «sensations de

1. E. Levinas, Carnets de captivité, in Carnets de captivité, suivi de Écrits sur la captivité et Notes philosophiques diverses (= Euvres 1), R. Calin et C. Chalier (éd.), Paris, Grasset - IMEC, 2009, p. 186. 
mouvement» aussi, que des champs kinesthésiques se donnent à moi. C'est par lui encore, mon corps charnel, que j'agis ou que je réagis sur le dehors. Le perçu accède ainsi à la conscience sous le mode du corporel comme remplissement originaire de l'intention par l'intuition donatrice.

2. Autrui : c'est de nouveau par ce corps que j'accède à autrui, à l'expérience d'autrui comme non originaire. Autrui en effet se présente d'abord dans mon champ de vision comme corps physique, puis selon l'intentionnalité singulière de l'Einfühlung, et c'est l'enseignement de la Cinquième Méditation cartésienne, comme tout autre chose, si je puis dire, qu'un simple objet. Autrui existe pour moi comme sujet percevant, comme sujet sentant, parce que je suis à même, comme Leib, de reconstituer, à partir de ses gestes, de ses mouvements physiques, leur image analogique dans mon corps. Les gestes d'autrui, les gestes qu'autrui effectue, éveillent des significations qui sont à la fois les siennes et les miennes. Ils suscitent en moi un écho, je peux intérieurement les mimer, et faire ainsi l'épreuve de ce que pourrait être la présence d'autrui. Sa vie intérieure, sa subjectivité corporelle, adviennent ainsi à partir de la mienne. Et s'il est possible qu'un dialogue ait lieu avec cet autre homme et qu'avec lui s'engage une véritable communauté de significations, en-deçà des mots et préparant l'usage du langage, c'est en vertu de l'expérience partagée en laquelle vivent tous les hommes, en vertu d'une référence commune dont notre existence corporelle est le lieu premier d'expérience. Si nous communiquons par les mots, c'est que nous appartenons déjà par le corps au même monde, avant les conventions du langage (je note en passant que cette structure de «l'Autre antérieur à la Parole» est rapportée par Levinas, avec toutes les contestations qu'on sait, au «Féminin $»^{2}$ ). Ainsi, incarné dans mon corps, dans et par cette incarnation, je suis un Je humain. La mise en évidence de mon corps propre signifie l'ouverture à l'essence spécifique du phénomène objectif Je en tant que cet homme-ci qui se présente devant moi.

3. Mon temps : cette centralité du corps-sujet, du corps sentant, détermine pareillement le temps comme mon temps, et la temporalité comme vécue. Non pas comme appréhension privée, subjective et solipsiste du temps, opposée à l'objectivité anonyme du temps des horloges et des calendriers mais comme extension dynamique du présent en présent vivant. Le présent vivant est praes-ens: ce qui est en avant de moi comme imminent. Le lieu du présent c'est mon corps, pourrait-on dire (d'ailleurs la langue commune le dit: j'étais présent, physiquement, sur les lieux). La pure présence des choses advient donc à partir d'une donation qui s'effectue au plus près

2. Notes philosophiques diverses, in Euvres 1, p. 269. 
des choses mêmes, c'est-à-dire telles qu'elles se donnent pour un corps percevant, dans la pure épreuve que fait le corps du monde environnant, dans une expérience vécue au présent qui est la forme même de la vie égologique. Il faudrait parler d'un « corps de conscience» pour qualifier ce corps qui vit les événements qui l'affectent beaucoup plus près des données sensorielles que ne le peut la conscience ${ }^{3}$. C'est parce que la conscience est supportée dans sa perceptivité même par un corps percevant qu'elle n'est jamais identique à elle-même, comme dit Husserl dans Ideen I, qu'elle est en quelque sorte une conscience qui aurait des pieds et tournerait autour de l'objet transcendant en en dégageant dans la succession la signification proprement temporelle, le côté res temporalis dont parlent les Ideen $I^{4}$.

Sous ces trois considérants brièvement rappelés, on pourrait dire que la phénoménologie husserlienne consiste à dégager une signification temporelle $d u$ corps selon une ligne temps-monde-corps tenue avec rigueur et d'ailleurs partagée ou reprise par Levinas lui-même sous bien des aspects, au point qu'elle parait souvent fournir un entêtant motif de fond du thème lévinassien. Son renversement en «signification corporelle du temps", selon le geste appliqué par l'auteur de Totalité et Infini à l'intentionnalité, décentre le propos et en bouleverse les articulations. Ce lien temps-monde-corps, Levinas le reçoit, le désintrique et le renoue autour d'Eros.

Pour Husserl, le corps constitue un événement où je coïncide avec moi, « expérienciellement» (erfahrungsmässig), selon une modification originaire dont il est le support et qui constitue l'élément natif d'une transcendance interne au champ temporel. Mais si, par mon corps percevant et incarné, une essence de l'homme, de son Je, vient au jour, «se révèle", puisque le corps-sujet, la chair, accède aux choses mêmes, si l'acquisition de la sphère absolue de l'être transcendantal doit se redoubler de son acquisition comme universalité intersubjective, on peut noter, et c'est décisif, qu'il n'y a pas ici, c'est-à-dire pour Husserl, de différence sexuelle. Ou plus exactement, la différence sexuelle est secondaire par rapport à l'incarnation, elle ne vient et ne peut jouer qu'après-coup, dans la sphère personnelle du Je.

3. Ibid.: «corps = conscience», «cette structure de corps = conscience», «cette position » qui permet l'ajournement de la jouissance, par le travail et la maison, «est corps » donc "elle est conscience», c'est-à-dire temps. Ce thème est continu et prégnant dans toute la période de Totalité et Infini. La structure corps = conscience est ce par quoi ma position naturelle sur terre sera mise en question par le «Féminin».

4. Idées directrices pour une phénoménologie, trad. fr. P. Ricœur, Paris, Gallimard, 1950, p. 502 : "la chose se donne en son essence idéale comme res temporalis, sous la "forme nécessaire du temps" - idéale au sens où l'idéation intuitive "nous révèle la chose comme durant nécessairement, susceptible d'une extension sans fin de durée». 
Or dans les textes du jeune Levinas, il n'y a de corps ou d'incarnation que sexué(e). On est là directement au cœur de la différence essentielle avec l'héritage phénoménologique husserlien. La «signification corporelle du temps» est, je le disais, comme la signature d'une note sur la caresse et l'instant. Elle désigne dans cette dizaine de lignes, même de façon un peu hâtive et elliptique, un lien, une reliaison entre «l'espoir pour le présent» et la «caresse». Ce lien se nomme, depuis les Carnets jusqu’à Totalité et Infini, Eros ou amour, de façon plus ou moins indifférenciée. Levinas écrit parfois «eros ${ }^{5}$ et entre parenthèses "amour» avec un point d'interrogation ${ }^{6}$. Lorsqu'il évoque les "sentiments indirects», souffrir de la souffrance de l'autre, se réjouir de sa joie ${ }^{7}$, il explique qu'ils assurent quelque chose d'un passage d'eros à amour ${ }^{8}$, ainsi distingués. Il y a une constante ambivalence dans les usages lévinassiens de l'équivalence amour-eros, dans le transport qui va de l'un à l'autre ou l'amalgame qui les fond en une unique matière. $\mathrm{La}$ «signification corporelle du temps» ressortit donc et fournit le concept clé des "contributions à l'analyse de l'eros" comme le dit Levinas lui-même à propos des notations qui émaillent les textes des Carnets sur ce point. Ces «contributions» diverses et inégales sont d'ailleurs regroupées sous un titre générique et philosophique («pour l'analyse de l'eros»), attestant ainsi de façon incontestable qu'il s'agit bien, avec cette analytique (phénoménologique?) de l'érotique, d'un projet qui court à travers les Carnets de captivité - projet anticipé dans De l'évasion et qui ira jusqu'à la "phénoménologie de l'eros» de Totalité et Infini. J’en dégagerai pour commencer quelques traits saillants.

Le premier, c'est ce qu'on pourrait appeler une «réduction érotique», c'est-à-dire au fond une reprise de la grande question pascalienne "m'aimet-on moi ? ». "L'ego ne se définit pas en dehors de l'amour chez moi » ${ }^{9}$, écrit Levinas, et il ajoute immédiatement "amour = sexualité» (selon l'ambivalente équivalence que je viens d'évoquer) car «sexualité constitutive de l'égoïté». Ceci constitue une détermination fondamentale. Il ne suffit donc pas que je sois, ni que je sois certain que je suis, que je veux, que j'agis, que je pense. Il faut encore, il faut premièrement que je sois aimé - pour être. Ainsi, l'événement fondamental de l'être, ce n'est pas l'être, c'est, pour reprendre une formule et tout le registre de De l'évasion, eros comme

5. Eros / eros / éros est orthographié plutôt avec une minuscule dans les Carnets et une majuscule dans Totalité et Infini, sans que cet usage général ne soit constamment transgressé par Levinas lui-même.

6. Notes philosophiques diverses, p. 441.

7. Carnets de captivité, p. 70.

8. Ibid., p. 99.

9. Ibid., p. 114 . 
«besoin d'évasion» hors de l'être, hors de «la suffisance du fait d'être ${ }^{10}$. Ce «besoin d'évasion» consiste à «s'écarter de ce qu'il y a de poids dans l'être», à "sortir». Sortir de l'être, c'est avant tout sortir de soi, de son être-rivé à soi. Je voudrais insister sur ce point. Le nom de cette sortie, c'est l'amour, le "vraiment-humain», comme le dit encore Levinas en $1985^{11}$. En effet, "Eros délivre de cet encombrement », comme le montrent encore les analyses de Totalité et Infini ${ }^{12}$. Dans les exemples d'évasion sur lesquels s'arrête le texte de 1935 (la nausée, la honte, l'être-mal-dans sa peau, etc.), le plaisir tient une place particulière. Je m'y arrête un instant car, plus encore que les autres cas d'évasion, il a un rapport immédiat avec le temps. Dans cette méditation radicalement non hédoniste du plaisir qu'on trouve dans De l'évasion, le plaisir est analysé comme ce par où l'être se vide de sa substance, s'allège, se dissémine. Levinas discute la thèse qui enracine le plaisir dans une concentration dans l'instant. Il y oppose une contre-thèse temporelle: dans le plaisir, écrit-il, l'instant est fractionné « dans un abandon, une perte de soi-même, une sortie en dehors de soi, une extase ${ }^{13}$. Telle est l'évasion érotique - une libération de l'être. Laquelle ne se peut que contre les métaphysiques qui «ravalent le plaisir au rang d'état et dissimulent le mouvement du plaisir dans lequel s'accomplit... la promesse d'évasion qu'il apporte au malaise du besoin ${ }^{14}$, contre les métaphysiques du vide et du plein, du manque et du monde, du besoin et du réel, et contre «les modèles de la satisfaction » évoqués bien plus tardivement et dans le même fil d'inspiration ${ }^{15}$, bref contre «la conception antique de l'amour» fils de poros et de penia $^{16}$ - laquelle ne permet pas de comprendre quoi que ce soit du «sexe», c'est la grande critique du jeune Levinas. Car «dans ma théorie de l'Eros, c'est le sexe qui devient la notion centrale ${ }^{17}$. Le plaisir brise les formes de l'être, comme dit De l'évasion, il est une "évasion" et cette évasion n'est pas chronologiquement ou logiquement subordonnée au besoin d'évasion. Elle le précède comme elle précède l'être lui-même. Le plaisir n'est pas dirigé vers l'accomplissement total de l'être limité, vers la dé-limitation et la satisfaction, mais "vers la délivrance et l'évasion ${ }^{18}$.

10. De l'évasion, Paris, Librairie générale française (Le Livre de poche), 1988, p. 106 et 93.

11. "Ce qui est vraiment humain, c'est - ne vous effrayez pas de ce mot - l'amour» (entretien avec Christoph von Wolzogen, trad. fr. A. David, Philosophie, ${ }^{\circ}$ 93, printemps 2007, p. 15).

12. Totalité et Infini, Paris, Librairie générale française (Le Livre de poche), 1990, p. 303 (dans tout le paragraphe intitulé «La subjectivité dans l'Eros»).

13. De l'évasion, p. 109.

14. Ibid.

15. De Dieu qui vient à l'idée, Paris, Vrin, 1992, p. 172.

16. Carnets de captivité, p. 114 et 117.

17. Ibid., p. 117.

18. De l'évasion, p. 120. 
Ce que découvre "pré-originellement» (pour user d'un anachronisme) l'amour, ce qu'il atteste immédiatement, c'est notre impossibilité première de demeurer rivés à nous-mêmes dans «l'expérience pure de l'être pur ${ }^{19}$. Telle est la signification de l'expression "l'événement de l'être». Un événement est une effectivité qui précède sa propre possibilité ou sa possibilisation ontologique comme passage à l'acte. C'est la raison pour laquelle Levinas note lapidairement dans ses Carnets de captivité: "Évasion dans quelque chose qui n'est pas être ${ }^{20}$, qui ne va certainement pas de l'être à l'être - et qui a pour nom "amour». "Cette catégorie de sortie», ajoute Levinas, n'est «jamais une sortie en dehors du temps ${ }^{21}$. On ne sort pas du temps, on s'insinue dans ses intervalles pour sortir de l'être. Sous cet aspect, Eros, en tant qu'il s'ouvre comme intervalle du temps (tout comme le besoin signifie un intervalle de l'espace ${ }^{22}$ ), suspend la présence de l'être et cette suspension est effectuée par le corps sexué ou par «l'impatience de la volupté » évoquée par Totalité et Infini ${ }^{23}$. Une catégorie du temps se tisse avec beaucoup de précision dans ce lien entre le sexe, le plaisir, la volupté et l'évasion et elle ne se peut qu'à partir de cette centralité du corps sexué soulignée dans les Carnets.

Le nœud par où le corps sexué, substitué au corps propre, au Leib asexué (car il y a bel et bien une physique du corps sexué, un corps physique parce que sexué) se présente comme une catégorie du temps, si l'on peut dire ainsi, c'est «la dualité insurmontable " ${ }^{24}$ que signifie Eros. D’ailleurs la translation du corps propre vers le corps sexué a des conséquences immédiatement temporelles. Au présent vivant se substitue, même si cela est moins clair chez le jeune Levinas, une exacerbation du temps dans l'instant. En tout état de cause, une des leçons de ces textes de jeunesse, c'est déjà qu'autrui et le corps d'autrui, «ça fait deux». L'érotique découpe le réel en duel ${ }^{25}$, selon une ligne qui n'autorise pas, malgré ce que suggère l'image, que les parties distribuées de part et d'autre de la ligne entrent en rapport l'une avec l'autre, en un rapport, donc, où le deux se relèverait et se réduirait à et dans l'un (genre / espèce, partie / tout, action / passion, vérité/ erreur, pour reprendre les exemples de la conférence de 1959). La

19. De l'évasion, p. 116.

20. Carnets de captivité, p. 175.

21. De l'évasion, p. 96-97 et 101.

22. Carnets de captivité, p. 129.

23. Totalité et Infini, p. 290 : «La volupté [...] va sans aller à une fin».

24. Carnets de captivité, p. 114 et 190.

25. E. Levinas, "Au-delà du possible» (conférence du 27 janvier 1959), in Parole et Silence et autres conférences inédites au Collège philosophique (= Euvres 2), R. Calin et C. Chalier (éd.), Paris, Grasset - IMEC, 2011, p. 311. 
sexualité, c'est par excellence le rapport avec l'absolument autre, c'est-àdire le rapport où la dualité précède et succède, encadre le rapport luimême qui n'est même plus un rapport. C'est la raison pour laquelle, s'il a à être médité en profondeur, l'amour devra préalablement s'excepter de «sa conception antique», c'est-à-dire qu'il lui faut s'affranchir de toute ontologie de l'un et de la fusion érotique et se confronter sans détour à la «dualité insurmontable de l'eros ${ }^{26}$. «Le fond même de l'amour» ( «la sexualité», ajoute immédiatement Levinas, et sans qu'il ne lui paraisse là encore nécessaire de revenir sur cette équivalence immédiate), c'est «la dualité et le mystère d'autrui» - ceci devant être rapporté à la phrase que j'indiquais en commençant: «l'ego ne se définit pas en-dehors de l'amour chez moi». L'ensemble formé par ces notations est tout à fait explicite: il s'agit de comprendre Eros en tant qu'il se "contredistingue » foncièrement de «la conception antique» ontologie-être-Un. «La sexualité comme origine du social ${ }^{27}$ ne peut s'entendre qu'à partir de la dualité, leitmotiv de tous ces passages du texte qui sont autant de glissements progressifs dans le texte. L'érotique est dit par ailleurs «source du temps humain»" ${ }^{28}$. En effet, la «dualité» indique une scansion significative du temps lui-même (sexualité/ société-socialité/ temporalité): «Lien dualité et le drame du temps s'éclaircira à partir de la dualité sociale c'est-à-dire sexuelle» ${ }^{29}$. La phrase est un peu gauche, bancale, et promet un éclaircissement plus qu'elle ne l'effectue, mais elle noue, me semble-t-il, ce qu'il en est de la «signification corporelle du temps» depuis la «dualité insurmontable» qui fait le fond de ce «drame du temps».

Je voudrais ici mentionner, sans le développer, un point de difficulté. Il y a, si on tente de lire ensemble ces textes de jeunesse, un nœud complexe et trouble entre l'être-rivé au corps qui caractérise «la philosophie de l'hitlérisme ${ }^{30}$, le besoin d'évasion qui en délivre et, malgré tout, le maintien, dans la prise en vue de la dualité d'eros, de l'impossibilité de sortir de son corps en dépit de la «trans-substantiation » érotique produite dans la volupté et la volupté de la voluptés1 ${ }^{1}$. 'équivoque érotique joue ici à plein. «L'hitlérisme» serait sans doute quelque chose comme une pensée du corps, à coup sûr, mais une pensée an-érotique $d u$ corps qui le viderait de toute équivoque temporelle, c'est-à-dire, au fond, de sa signification corporelle la plus marquée - une pensée du corps qui raterait le corps en

26. Carnets de captivité, p. 114 et 190.

27. Ibid., p. 66.

28. "Au-delà du possible», p. 311.

29. Carnets de captivité, p. 66.

30. Quelques réflexions sur la philosophie de l'hitlérisme, Paris, Rivages, 1997.

31. Totalité et Infini, p. 298. 
en ratant la temporalité. En effet le jeu, si je puis dire, de l'être-rivé et de l'évasion, c'est le temps, la temporalité comme "intervalle» ou comme séparation d'avec le désirable ${ }^{32}$. Les analyses du temps dans les Carnets et dans la phénoménologie du plaisir de De l'évasion mettent clairement en évidence la fonction désidentifiante de l'instant, cette non-coïncidence absolue de moi avec moi dans l'instant érotique, ce que Levinas appelle dans la note sur la signification corporelle du temps, comme dans plusieurs autres notes ${ }^{33}$, l' «espoir pour l'instant» (ou pour le présent, ce qui vaudrait à la fois pour la caresse et pour la foi). L'instant «crie vengeance» ${ }^{34}$. Il appelle à une réparation dans l'instant, au présent, pour une imminence déjà éthique qui ne supporte aucun diffèrement. Dans ce «cri» du temps instantané se tiendrait en quelque sorte la vérité de la temporalité ellemême, dès lors qu'elle est engagée, et elle l'est toujours-déjà, évidemment, dans la multiplicité de ses équivoques. C'est d'ailleurs à ce titre que le sexe constitue «la base» de tout rapport social, de toute dualité érotique (ou, plus tard, éthique, je m'empresse de l'ajouter). On ne tiendra donc jamais assez compte du «sexe». Freud, par exemple, est accusé de sous-estimer la sexualité ${ }^{35}$ puisqu'il ne va pas, selon Levinas, jusqu'à y discerner la source du temps humain.

On comprend pourquoi la lecture de Proust qu'attestent les Carnets détient une importance cruciale. On n'en évalue jamais mieux la portée que lorsque Hegel se substitue à Proust, dans une certaine mesure, comme dans ce passage de la conférence du 27 janvier 1959, déjà citée, où l'instant érotique et la durée de la paternité sont dialectiquement opposés puis réconciliés dans la merveille humaine de la famille ${ }^{36}$ - ce qui place le lecteur, me semble-t-il, devant de considérables difficultés. Proust donc. La «signification corporelle du temps» pourrait très bien désigner en effet et l'entreprise proustienne, la qualité propre de l'écriture du temps qu'elle engage, et caractériser en bonne partie l'œuvre lévinassienne au moins jusqu'à Totalité et Infini. Le temps perdu et retrouvé et l'éthique et sa vérité en plusieurs temps, selon une formule plus tardive, se répondent. L'amour et le temps, le face-à-face et la sensibilité, l'invisible et le corps, la transcendance et la peau nue, cette nudité elle-même, comme telle, érotique, et la nudité ou la fragilité, éthique, qu'elle expose s'entre-signifient. Cette «entr'implication» me paraît essentielle pour comprendre la double intrigue

32. Notes philosophiques diverses, p. 279.

33. Ibid., p. 407 par exemple.

34. Ibid., p. 462.

35. Ibid., p. 434.

36. «Au-delà du possible», p. 311. 
de l'érotique et de l'éthique selon cette dualité inassimilable qui marque asymétriquement la temporalité du face-à-face éthique. La «signification corporelle du temps» contient et excède la signification temporelle du corps - et il faut voir dans cette "excession", me semble-t-il, la puissance et la fécondité de l'équivoque dans la pensée de Levinas, dans son cœur et son ressort le plus enfoui.

Un dernier point, quasi conclusif. Dans toutes ces approches phénoménologiques d'Eros, de la caresse, du plaisir, de la volupté, dans les Carnets ou dans De l'évasion et jusqu'à Totalité et Infini (peut-être même au-delà, mais je n'entrerai pas ici dans un examen chrono-comparatif), Levinas se montre très attentif au risque que l'amour ne se dégrade en amour de l'amour, en un sentiment intentionnel ou ré-intentionnalisé par le de, qu'il finisse en un retour à soi après avoir suscité l'évasion hors de son enfermement en soi. Cette attention le conduit à remarquer qu'il ne s'agit en aucun cas d'une intention ou d'un égoïsme. Le sujet de la «signification corporelle du temps» du plaisir est le soi d'un autre, il n'est pas le soi de soi-même - d'ailleurs, le temps, c'est exactement cela: le soi-même n'est pas le même soi, le temps a passé! Si ce n'était pas le cas, l'équivoque ne pourrait se maintenir, elle virerait très vite en univoque intentionnelle. On peut aller jusqu'à dire que, chez Levinas, l'équivoque, c'est le temps. Il y a là, autour des variations, parfois considérables, de la position lévinassienne sur l'amour (sur lesquelles je n'ai pas voulu insister), une sorte d'arc de pensée qui me paraît d'une grande pertinence. Le «vraiment-humain» de l'amour, dans la diversité et la multiplicité de ses modulations dans l'écriture lévinassienne, amènerait à scruter plus qu'on ne l'a fait jusqu'à présent la façon dont s'effectuent chez Levinas les "passages» de l'érotique à l'éthique, de l'éthique à la politique, de l'éthique à la morale, le poids de l'ontologique lié à l'éthique dans le mouvement même de leur dé-solidarisation et de leur fluidification. Ces «passages» ne sont pas des passages, toute la difficulté est là. Ce sont des intrigues et des interruptions, qui transportent aussi avec elles ce qu'elles séparent et arrêtent, sans pour autant s'abîmer en dialectique.

Éthique et amour se nouent tous deux temporellement autour de l'unicité de l'autre et de l'unicité du soi devant l'autre. Je n'ignore nullement les profondes démarcations, les contre-distinctions, les oppositions que souligne Levinas entre les deux registres. Il m'a semblé cependant qu'on pouvait les tenir ensemble dans la signification originairement corporelle de la temporalité, soit comme indices différenciés de cette signification. En tout état de cause, on aura beau insister sur leurs évidentes différences, il ne me parait guère possible, en raison de l'ouverture heuristique emportée par ce concept de «signification corporelle du temps», de penser l'érotique comme le non-éthique, pas plus que l'éthique n'en peut être la simple relève. 
Car la phénoménologie de l'Eros n'appartient pas à la jouissance élémentale (y compris dans l'économie générale de Totalité et Infini). Son mouvement, Levinas le nomme profanation. Cette profanation est une révélation, une révélation de l'éthique. Mais cette révélation ne s'avère que dans l'équivoque de la profanation. L'équivoque de l'érotique, c'est que l'érotique ne se réduit jamais à l'érotique, son mouvement le porte incessamment au-delà de lui-même et de la simple réalisation de ses possibilités ${ }^{37}$. L'équivoque est équivoque du temps. Et il n'est pas interdit de présumer qu'on peut en étendre la portée bien au-delà de l'érotique. L'équivoque temporelle régit les passages, les interruptions, les transitions entre les «stades» (J. Rolland proposait de reprendre la notion kierkegaardienne pour penser le "saut» de l'érotique à l'éthique). Une semblable équivoque temporelle se joue également entre proximité et justice. Et pour aller au bout de cette hypothèse, il faudrait envisager une possible équivoque de l'éthique dès lors qu'elle a à entendre le «cri» des tiers d'un côté, la politique qui vient après elle, et l'envisagement amoureux de l'autre, qui la concurrence d'une certaine façon. L'équivoque de l'éthique se nouerait alors dans ce qui, d'elle, se vide et se nourrit à la fois d'Eros et de Polis, de Hessed et de Tsedaka. Une éthique de la «signification corporelle du temps» pourrait ainsi se comprendre comme une éthique de la temporalisation interminable de la subjectivité humaine, c'est-à-dire du "vraiment-humain», du non-synthétisable ou du

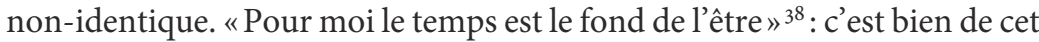
ancrage que le désir tire sa vie même, soit son excendance, comme disait $D e$ l'évasion. L'amour, y compris et surtout dans sa charge sexuée et sexuelle, constitue le thème qui seul peut en vérité faire pièce à l'ontologie - voilà le premier trait d'où le philosopher lévinassien se sera mis spontanément en branle.

Gérard Bensussan

Université de Strasbourg

37. Cf. Totalité et Infini, p. 292: «L'éros ne s'accomplit pas comme un sujet qui fixe un objet, ni comme une pro-jection, vers un possible. Son mouvement consiste à aller au-delà du possible».

38. Carnets de captivité, p. 134. 